Acta Crystallographica Section E

Structure Reports

Online

ISSN 1600-5368

\section{5-Bromo-3-(4-fluorophenylsulfonyl)-2,7- dimethyl-1-benzofuran}

\section{Hong Dae Choi, ${ }^{\text {a }}$ Pil Ja Seo ${ }^{a}$ and Uk Lee ${ }^{b *}$}

aDepartment of Chemistry, Dongeui University, San 24 Kaya-dong, Busanjin-gu, Busan 614-714, Republic of Korea, and ${ }^{\mathbf{b}}$ Department of Chemistry, Pukyong National University, 599-1 Daeyeon 3-dong, Nam-gu, Busan 608-737, Republic of Korea

Correspondence e-mail: uklee@pknu.ac.kr

Received 15 October 2012; accepted 21 October 2012

Key indicators: single-crystal X-ray study; $T=173 \mathrm{~K}$; mean $\sigma(\mathrm{C}-\mathrm{C})=0.003 \AA$; $R$ factor $=0.028 ; w R$ factor $=0.074 ;$ data-to-parameter ratio $=18.3$.

In the title compound, $\mathrm{C}_{16} \mathrm{H}_{12} \mathrm{BrFO}_{3} \mathrm{~S}$, the 4-fluorophenyl ring makes a dihedral angle of $72.35(5)^{\circ}$ with the mean plane [r.m.s. deviation $=0.008$ (1) $\AA$ ] of the benzofuran fragment. In the crystal, molecules are linked into [010] chains via two different inversion-generated pairs of $\mathrm{C}-\mathrm{H} \cdots \mathrm{O}$ hydrogen bonds. The crystal structure also exhibits slipped $\pi-\pi$ interactions between the benzene rings of neighbouring molecules [centroid-centroid distance $=3.667$ (2) $\AA$ and slippage = 1.341 (2) $\AA$ ], and between the benzene and the furan rings of neighbouring molecules [centroid-centroid distance = 3.759 (2) $\AA$ and slippage $=0.757$ (2) $\mathrm{A}]$.

\section{Related literature}

For background information and the crystal structures of related compounds, see: Choi et al. (2010, 2012).<smiles>Cc1oc2c(C)cc(Br)cc2c1S(=O)(=O)c1ccc(F)cc1</smiles>

\section{Experimental}

Crystal data

$\mathrm{C}_{16} \mathrm{H}_{12} \mathrm{BrFO}_{3} \mathrm{~S}$

$M_{r}=383.23$

Triclinic, $P \overline{1}$

$a=8.0789$ (1) ^

$b=9.2754(2) \AA$

$c=11.3555(2) \AA$

$\alpha=71.283$ (1)

$\beta=72.645(1)^{\circ}$

$$
\begin{aligned}
& \gamma=70.042(1)^{\circ} \\
& V=740.30(2) \AA^{3} \\
& Z=2 \\
& \text { Mo } K \alpha \text { radiation } \\
& \mu=2.94 \mathrm{~mm}^{-1} \\
& T=173 \mathrm{~K} \\
& 0.29 \times 0.21 \times 0.18 \mathrm{~mm}
\end{aligned}
$$

\section{Data collection}

Bruker SMART APEXII CCD diffractometer

Absorption correction: multi-scan (SADABS; Bruker, 2009)

$T_{\min }=0.527, T_{\max }=0.746$

Refinement

$R\left[F^{2}>2 \sigma\left(F^{2}\right)\right]=0.028$

$w R\left(F^{2}\right)=0.074$

$S=1.02$

3680 reflections

201 parameters

$\mathrm{H}$-atom parameters constrained

$\Delta \rho_{\max }=0.36 \mathrm{e}^{-3}$

$\Delta \rho_{\min }=-0.47$ e $\AA^{-3}$

Table 1

Hydrogen-bond geometry $\left(\AA{ }^{\circ}\right)$.

\begin{tabular}{lllll}
\hline$D-\mathrm{H} \cdots A$ & $D-\mathrm{H}$ & $\mathrm{H} \cdots A$ & $D \cdots A$ & $D-\mathrm{H} \cdots A$ \\
\hline $\mathrm{C} 3-\mathrm{H} 3 \cdots \mathrm{O} 2^{\mathrm{i}}$ & 0.95 & 2.53 & $3.408(2)$ & 154 \\
$\mathrm{C} 9-\mathrm{H} 9 A \cdots \mathrm{O} 2^{\mathrm{ii}}$ & 0.98 & 2.50 & $3.304(2)$ & 139 \\
\hline
\end{tabular}

Symmetry codes: (i) $-x+1,-y+1,-z+1$; (ii) $-x+1,-y,-z+1$.

Data collection: APEX2 (Bruker, 2009); cell refinement: SAINT (Bruker, 2009); data reduction: $S A I N T$; $\operatorname{program}(\mathrm{s})$ used to solve structure: SHELXS97 (Sheldrick, 2008); program(s) used to refine structure: SHELXL97 (Sheldrick, 2008); molecular graphics: ORTEP-3 (Farrugia, 1997) and DIAMOND (Brandenburg, 1998); software used to prepare material for publication: SHELXL97.

This work was supported by the Blue-Bio Industry Regional Innovation Center (RIC08-06-07) at Dongeui University as an RIC program under the Ministry of Knowledge Economy and Busan City.

Supplementary data and figures for this paper are available from the IUCr electronic archives (Reference: HB6977).

\title{
References
}

Brandenburg, K. (1998). DIAMOND. Crystal Impact GbR, Bonn, Germany. Bruker (2009). APEX2, SADABS and SAINT. Bruker AXS Inc., Madison, Wisconsin, USA.

Choi, H. D., Seo, P. J. \& Lee, U. (2012). Acta Cryst. E68, o2143.

Choi, H. D., Seo, P. J., Son, B. W. \& Lee, U. (2010). Acta Cryst. E66, o2049.

Farrugia, L. J. (1997). J. Appl. Cryst. 30, 565.

Sheldrick, G. M. (2008). Acta Cryst. A64, 112-122. 


\section{supporting information}

Acta Cryst. (2012). E68, o3208 [doi:10.1107/S1600536812043607]

\section{5-Bromo-3-(4-fluorophenylsulfonyl)-2,7-dimethyl-1-benzofuran}

\section{Hong Dae Choi, Pil Ja Seo and Uk Lee}

\section{S1. Comment}

As a part of our continuing study of 5-bromo-2-methyl-1-benzofuran derivatives containing 4-fluorophenylsulfonyl (Choi et al., 2010) and 4-methylphenylsulfonyl (Choi et al., 2012) substituents in 3-position, we report herein the crystal structure of the title compound.

In the title molecule (Fig. 1), the benzofuran unit is essentially planar, with a mean deviation of 0.008 (1) $\AA$ from the least-squares plane defined by the nine constituent atoms. The dihedral angle between the 4-fluorophenyl ring and the mean plane of the benzofuran ring is 72.35 (5). In the crystal structure (Fig. 2), molecules are linked via pairs of C$\mathrm{H} \cdots \mathrm{O}$ hydrogen bonds (Table 1), forming inversion dimers. The crystal packing (Fig. 2) also exhibits slipped $\pi-\pi$ interactions; the first one between the benzene rings of neighbouring molecules, with a $\mathrm{Cg} 1{ }^{\cdots} \mathrm{Cg} 1{ }^{\mathrm{iii}}$ distance of 3.667 (2) $\AA$ and an interplanar distance of 3.413 (2) $\AA$ resulting in a slippage of 1.341 (2) $\AA$ ( $\mathrm{Cg} 1$ is the centroid of the $\mathrm{C} 2-\mathrm{C} 7$ benzene ring), and the second one between the benzene and the furan rings of neighbouring molecules, with a $\mathrm{Cg} 1{ }^{\cdots} \mathrm{Cg} 2^{\mathrm{ii}}$ distance of 3.759 (2) $\AA$ and an interplanar distance of 3.682 (2) $\AA$ resulting in a slippage of 0.757 (2) $\AA$ (Cg2 is the $\mathrm{C} 1 / \mathrm{C} 2 / \mathrm{C} 7 / \mathrm{O} 1 / \mathrm{C} 8$ furan ring).

\section{S2. Experimental}

3-Chloroperoxybenzoic acid (77\%, $381 \mathrm{mg}, 1.7 \mathrm{mmol})$ was added in small portions to a stirred solution of 5-bromo-3-(4fluorophenylsulfanyl)-2,7-dimethyl-1-benzofuran $(281 \mathrm{mg}, 0.8 \mathrm{mmol})$ in dichloromethane $(40 \mathrm{ml})$ at $273 \mathrm{~K}$. After being stirred at room temperature for $10 \mathrm{~h}$, the mixture was washed with saturated sodium bicarbonate solution and the organic layer was separated, dried over magnesium sulfate, filtered and concentrated at reduced pressure. The residue was purified by column chromatography (hexane-ethyl acetate, $4: 1 \mathrm{v} / \mathrm{v}$ ) to afford the title compound as a colorless solid [yield 71\%, m.p. 472-473 K; $R_{\mathrm{f}}=0.57$ (hexane-ethyl acetate, $4: 1 \mathrm{v} / \mathrm{v}$ )]. Colourless blocks were prepared by slow evaporation of a solution of the title compound in ethyl acetate at room temperature.

\section{S3. Refinement}

All $\mathrm{H}$ atoms were positioned geometrically and refined using a riding model, with $\mathrm{C}-\mathrm{H}=0.95 \AA$ for aryl and $0.98 \AA$ for methyl $\mathrm{H}$ atoms. $U_{\text {iso }}(\mathrm{H})=1.2 U_{\mathrm{eq}}(\mathrm{C})$ for aryl and $1.5 U_{\mathrm{eq}}(\mathrm{C})$ for methyl $\mathrm{H}$ atoms. The positions of methyl hydrogens were optimized rotationally. 


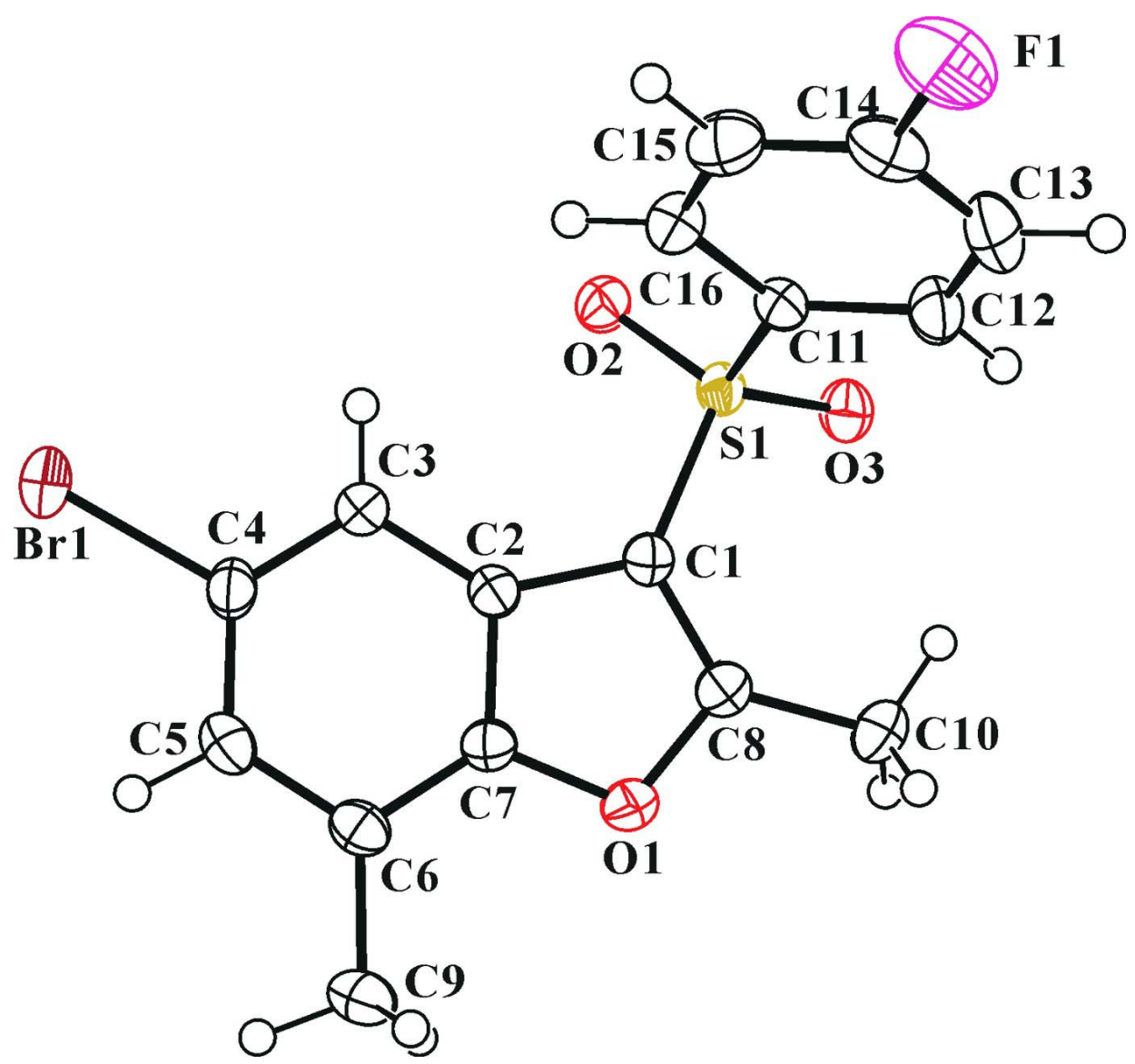

\section{Figure 1}

The molecular structure of the title compound with displacement ellipsoids drawn at the $50 \%$ probability level. $\mathrm{H}$ atoms are presented as small spheres of arbitrary radius. 


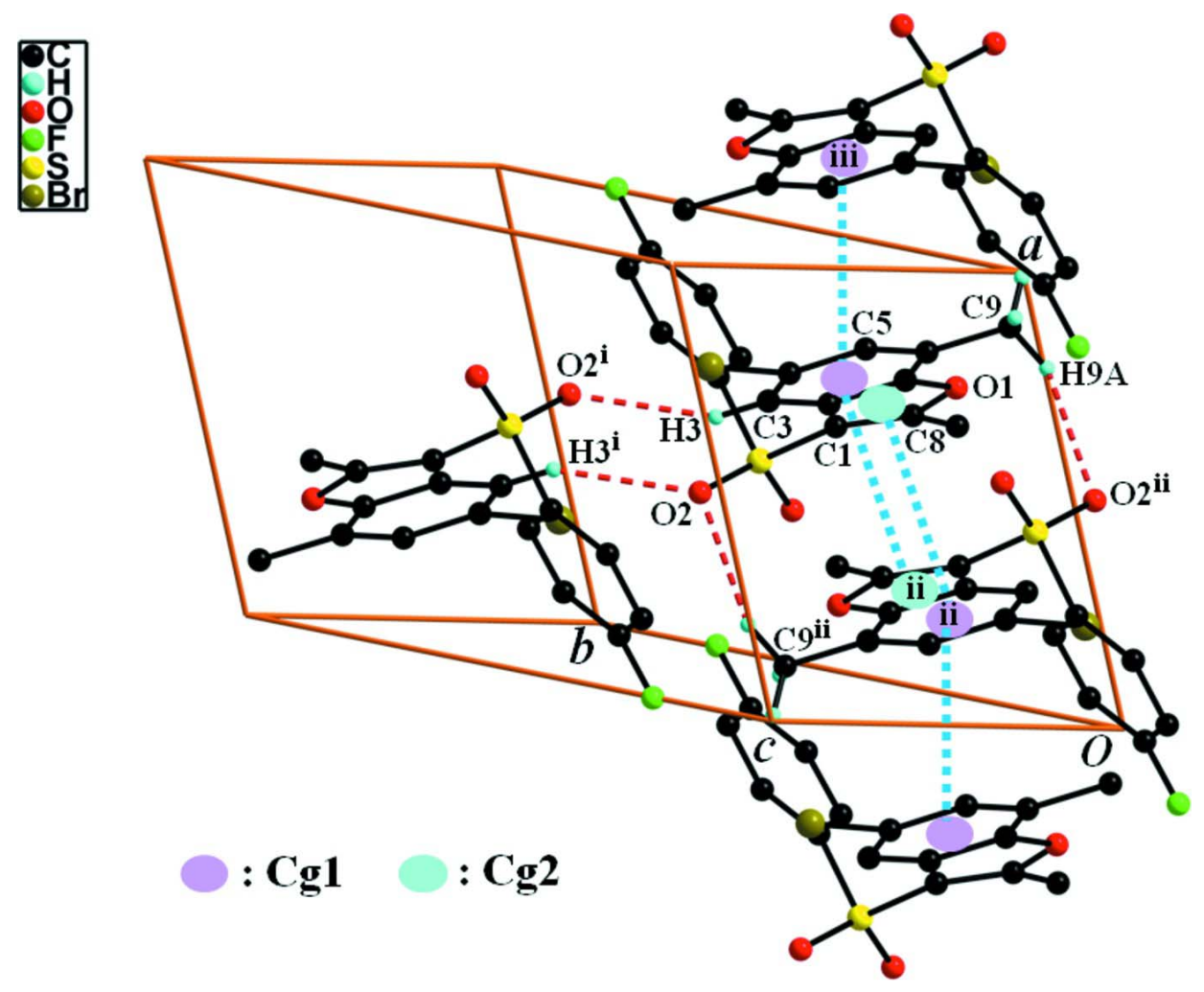

\section{Figure 2}

A view of the $\mathrm{C}-\mathrm{H} \cdots \mathrm{O}$ and $\pi-\pi$ interactions (dotted lines) in the crystal structure of the title compound. $\mathrm{H}$ atoms nonparticipating in hydrogen-bonding were omitted for clarity. [Symmetry codes: (i) $-x+1,-y+1,-z+1$; (ii) $-x+1,-y,-$ $z+1 ;$ (iii) $-x+2,-y,-z+1$.]

\section{5-Bromo-3-(4-fluorophenylsulfonyl)-2,7-dimethyl-1-benzofuran}

\section{Crystal data}

$\mathrm{C}_{16} \mathrm{H}_{12} \mathrm{BrFO}_{3} \mathrm{~S}$

$M_{r}=383.23$

Triclinic, $P \overline{1}$

Hall symbol: -P 1

$a=8.0789$ (1) $\AA$

$b=9.2754(2) \AA$

$c=11.3555(2) \AA$

$\alpha=71.283(1)^{\circ}$

$\beta=72.645(1)^{\circ}$

$\gamma=70.042(1)^{\circ}$

$V=740.30(2) \AA^{3}$

\section{Data collection}

Bruker SMART APEXII CCD diffractometer

Radiation source: rotating anode Graphite multilayer monochromator Detector resolution: 10.0 pixels $\mathrm{mm}^{-1}$ $\varphi$ and $\omega$ scans
$Z=2$

$F(000)=384$

$D_{\mathrm{x}}=1.719 \mathrm{Mg} \mathrm{m}^{-3}$

Melting point $=472-473 \mathrm{~K}$

Mo $K \alpha$ radiation, $\lambda=0.71073 \AA$

Cell parameters from 6655 reflections

$\theta=2.7-28.3^{\circ}$

$\mu=2.94 \mathrm{~mm}^{-1}$

$T=173 \mathrm{~K}$

Block, colourless

$0.29 \times 0.21 \times 0.18 \mathrm{~mm}$

Absorption correction: multi-scan

(SADABS; Bruker, 2009)

$T_{\min }=0.527, T_{\max }=0.746$

14090 measured reflections

3680 independent reflections

3259 reflections with $I>2 \sigma(I)$

$R_{\text {int }}=0.031$ 
$\theta_{\text {max }}=28.3^{\circ}, \theta_{\text {min }}=1.9^{\circ}$

$h=-10 \rightarrow 10$

Refinement

Refinement on $F^{2}$

Least-squares matrix: full

$R\left[F^{2}>2 \sigma\left(F^{2}\right)\right]=0.028$

$w R\left(F^{2}\right)=0.074$

$S=1.02$

3680 reflections

201 parameters

0 restraints

Primary atom site location: structure-invariant direct methods $k=-12 \rightarrow 12$

$l=-15 \rightarrow 15$

Secondary atom site location: difference Fourier map

Hydrogen site location: difference Fourier map

$\mathrm{H}$-atom parameters constrained

$w=1 /\left[\sigma^{2}\left(F_{\mathrm{o}}^{2}\right)+(0.0417 P)^{2}+0.237 P\right]$

where $P=\left(F_{\mathrm{o}}^{2}+2 F_{\mathrm{c}}{ }^{2}\right) / 3$

$(\Delta / \sigma)_{\max }=0.002$

$\Delta \rho_{\max }=0.36 \mathrm{e} \AA^{-3}$

$\Delta \rho_{\min }=-0.47$ e $\AA^{-3}$

\section{Special details}

Geometry. All esds (except the esd in the dihedral angle between two 1.s. planes) are estimated using the full covariance matrix. The cell esds are taken into account individually in the estimation of esds in distances, angles and torsion angles; correlations between esds in cell parameters are only used when they are defined by crystal symmetry. An approximate (isotropic) treatment of cell esds is used for estimating esds involving l.s. planes.

Refinement. Refinement of $\mathrm{F}^{2}$ against ALL reflections. The weighted R-factor $\mathrm{wR}$ and goodness of fit $\mathrm{S}$ are based on $\mathrm{F}^{2}$, conventional R-factors $R$ are based on $F$, with $F$ set to zero for negative $F^{2}$. The threshold expression of $F^{2}>2 \operatorname{sigma}\left(F^{2}\right)$ is used only for calculating R-factors(gt) etc. and is not relevant to the choice of reflections for refinement. R-factors based on $\mathrm{F}^{2}$ are statistically about twice as large as those based on F, and R- factors based on ALL data will be even larger.

Fractional atomic coordinates and isotropic or equivalent isotropic displacement parameters $\left(\AA^{2}\right)$

\begin{tabular}{|c|c|c|c|c|}
\hline & $x$ & $y$ & $z$ & $U_{\text {iso }} * / U_{\text {eq }}$ \\
\hline $\mathrm{Br} 1$ & $0.75349(3)$ & $0.10966(3)$ & $0.797351(17)$ & $0.03912(8)$ \\
\hline $\mathrm{S} 1$ & $0.48725(5)$ & $0.40486(5)$ & $0.29104(4)$ & $0.02342(10)$ \\
\hline F1 & 1.00247 (19) & $0.77090(18)$ & $0.00357(14)$ & $0.0571(4)$ \\
\hline $\mathrm{O} 1$ & 0.75109 (17) & $-0.04433(15)$ & $0.32764(12)$ & $0.0274(3)$ \\
\hline $\mathrm{O} 2$ & $0.40458(16)$ & $0.45572(16)$ & $0.40738(11)$ & 0.0286 \\
\hline $\mathrm{O} 3$ & 0.37705 (17) & $0.41993(16)$ & $0.20642(12)$ & 0.0315 \\
\hline $\mathrm{C} 1$ & $0.6075(2)$ & $0.2081(2)$ & $0.33360(16)$ & $0.0242(3)$ \\
\hline $\mathrm{C} 2$ & $0.6733(2)$ & $0.1303(2)$ & $0.45008(15)$ & 0.0227 \\
\hline $\mathrm{C} 3$ & $0.6674(2)$ & $0.1745(2)$ & $0.55829(16)$ & $0.0251(3)$ \\
\hline H3 & 0.6078 & 0.2785 & 0.5692 & $0.030^{*}$ \\
\hline $\mathrm{C} 4$ & $0.7538(2)$ & $0.0574(2)$ & $0.64843(16)$ & 0.0271 \\
\hline $\mathrm{C} 5$ & $0.8430(2)$ & $-0.0963(2)$ & $0.63592(17)$ & $0.0285(4)$ \\
\hline H5 & 0.9010 & -0.1707 & 0.7011 & $0.034^{*}$ \\
\hline C6 & $0.8486(2)$ & $-0.1426(2)$ & $0.52969(17)$ & 0.0268 \\
\hline $\mathrm{C} 7$ & $0.7611(2)$ & $-0.0248(2)$ & $0.44028(16)$ & 0.0243 \\
\hline $\mathrm{C} 8$ & $0.6581(2)$ & $0.0987(2)$ & $0.26428(16)$ & 0.0265 \\
\hline $\mathrm{C} 9$ & $0.9424(3)$ & $-0.3065(2)$ & 0.51205 (19) & $0.0331(4)$ \\
\hline H9A & 0.8612 & -0.3460 & 0.4891 & $0.050 *$ \\
\hline H9B & 0.9767 & -0.3761 & 0.5915 & $0.050 *$ \\
\hline H9C & 1.0506 & -0.3045 & 0.4440 & $0.050 *$ \\
\hline $\mathrm{C} 10$ & $0.6362(3)$ & 0.1018 & $0.13857(18)$ & $0.0346(4)$ \\
\hline $\mathrm{H} 10 \mathrm{~A}$ & 0.5784 & 0.2103 & 0.0967 & $0.052 *$ \\
\hline H10B & 0.5610 & 0.0329 & 0.1499 & $0.052 *$ \\
\hline
\end{tabular}


supporting information

$\begin{array}{lllll}\text { H10C } & 0.7547 & 0.0644 & 0.0858 & 0.052^{*} \\ \text { C11 } & 0.6478(2) & 0.5107(2) & 0.20442(16) & 0.0244(3) \\ \text { C12 } & 0.6821(3) & 0.5500(2) & 0.07255(17) & 0.0321(4) \\ \text { H12 } & 0.6237 & 0.5157 & 0.0295 & 0.038^{*} \\ \text { C13 } & 0.8014(3) & 0.6392(3) & 0.00442(19) & 0.0394(5) \\ \text { H13 } & 0.8256 & 0.6685 & -0.0859 & 0.047^{*} \\ \text { C14 } & 0.8840(3) & 0.6845(2) & 0.0705(2) & 0.0376(4) \\ \text { C15 } & 0.8550(3) & 0.6459(3) & 0.2007(2) & 0.0370(4) \\ \text { H15 } & 0.9169 & 0.6782 & 0.2427 & 0.044^{*} \\ \text { C16 } & 0.7330(2) & 0.5585(2) & 0.26933(17) & 0.0300(4) \\ \text { H16 } & 0.7078 & 0.5316 & 0.3597 & 0.036^{*}\end{array}$

Atomic displacement parameters $\left(\AA^{2}\right)$

\begin{tabular}{lllllll}
\hline & $U^{11}$ & $U^{22}$ & $U^{33}$ & $U^{12}$ & $U^{13}$ & $U^{23}$ \\
\hline Br1 & $0.05355(14)$ & $0.04150(14)$ & $0.02493(11)$ & $-0.01030(10)$ & $-0.01583(8)$ & $-0.00744(8)$ \\
S1 & $0.02474(19)$ & $0.0241(2)$ & $0.02165(19)$ & $-0.00430(16)$ & $-0.00808(15)$ & $-0.00546(16)$ \\
F1 & $0.0558(8)$ & $0.0497(9)$ & $0.0620(9)$ & $-0.0322(7)$ & $-0.0040(7)$ & $0.0026(7)$ \\
O1 & $0.0325(6)$ & $0.0237(6)$ & $0.0290(6)$ & $-0.0070(5)$ & $-0.0088(5)$ & $-0.0090(5)$ \\
O2 & $0.0296(6)$ & $0.0290(7)$ & $0.0250(6)$ & $-0.0048(5)$ & $-0.0041(5)$ & $-0.0089(5)$ \\
O3 & $0.0325(6)$ & $0.0346(7)$ & $0.0306(6)$ & $-0.0073(6)$ & $-0.0154(5)$ & $-0.0061(6)$ \\
C1 & $0.0276(8)$ & $0.0232(8)$ & $0.0228(7)$ & $-0.0067(7)$ & $-0.0077(6)$ & $-0.0049(7)$ \\
C2 & $0.0230(7)$ & $0.0222(8)$ & $0.0226(7)$ & $-0.0064(6)$ & $-0.0058(6)$ & $-0.0041(6)$ \\
C3 & $0.0285(8)$ & $0.0234(9)$ & $0.0237(8)$ & $-0.0065(7)$ & $-0.0063(6)$ & $-0.0059(7)$ \\
C4 & $0.0319(8)$ & $0.0303(9)$ & $0.0201(7)$ & $-0.0097(7)$ & $-0.0074(6)$ & $-0.0045(7)$ \\
C5 & $0.0305(8)$ & $0.0260(9)$ & $0.0265(8)$ & $-0.0090(7)$ & $-0.0089(7)$ & $0.0009(7)$ \\
C6 & $0.0271(8)$ & $0.0224(9)$ & $0.0294(8)$ & $-0.0091(7)$ & $-0.0058(7)$ & $-0.0020(7)$ \\
C7 & $0.0262(8)$ & $0.0226(9)$ & $0.0253(8)$ & $-0.0075(7)$ & $-0.0055(6)$ & $-0.0061(7)$ \\
C8 & $0.0277(8)$ & $0.0264(9)$ & $0.0267(8)$ & $-0.0077(7)$ & $-0.0062(6)$ & $-0.0075(7)$ \\
C9 & $0.0357(9)$ & $0.0217(9)$ & $0.0397(10)$ & $-0.0056(8)$ & $-0.0106(8)$ & $-0.0047(8)$ \\
C10 & $0.0431(10)$ & $0.0364(11)$ & $0.0302(9)$ & $-0.0093(9)$ & $-0.0125(8)$ & $-0.0132(8)$ \\
C11 & $0.0267(8)$ & $0.0208(8)$ & $0.0235(8)$ & $-0.0034(7)$ & $-0.0072(6)$ & $-0.0041(7)$ \\
C12 & $0.0360(9)$ & $0.0344(10)$ & $0.0253(8)$ & $-0.0077(8)$ & $-0.0113(7)$ & $-0.0041(8)$ \\
C13 & $0.0435(11)$ & $0.0415(12)$ & $0.0254(9)$ & $-0.0122(9)$ & $-0.0071(8)$ & $0.0027(8)$ \\
C14 & $0.0367(10)$ & $0.0264(10)$ & $0.0437(11)$ & $-0.0116(8)$ & $-0.0042(8)$ & $-0.0013(9)$ \\
C15 & $0.0387(10)$ & $0.0345(11)$ & $0.0445(11)$ & $-0.0135(9)$ & $-0.0100(8)$ & $-0.0135(9)$ \\
C16 & $0.0343(9)$ & $0.0305(10)$ & $0.0268(8)$ & $-0.0077(8)$ & $-0.0078(7)$ & $-0.0089(8)$ \\
& & & & & & \\
\hline & & & & & &
\end{tabular}

Geometric parameters ( $\left.\AA,{ }^{\circ}\right)$

\begin{tabular}{llll}
\hline $\mathrm{Br} 1-\mathrm{C} 4$ & $1.9019(16)$ & $\mathrm{C} 6-\mathrm{C} 9$ & $1.498(3)$ \\
$\mathrm{S} 1-\mathrm{O} 2$ & $1.4396(12)$ & $\mathrm{C} 8-\mathrm{C} 10$ & $1.480(2)$ \\
$\mathrm{S} 1-\mathrm{O} 3$ & $1.4403(12)$ & $\mathrm{C} 9-\mathrm{H} 9 \mathrm{~A}$ & 0.9800 \\
$\mathrm{~S} 1-\mathrm{C} 1$ & $1.7354(18)$ & $\mathrm{C} 9-\mathrm{H} 9 \mathrm{~B}$ & 0.9800 \\
$\mathrm{~S} 1-\mathrm{C} 11$ & $1.7612(17)$ & $\mathrm{C} 9-\mathrm{H} 9 \mathrm{C}$ & 0.9800 \\
$\mathrm{~F} 1-\mathrm{C} 14$ & $1.354(2)$ & $\mathrm{C} 10-\mathrm{H} 10 \mathrm{~A}$ & 0.9800 \\
$\mathrm{O} 1-\mathrm{C} 8$ & $1.368(2)$ & $\mathrm{C} 10-\mathrm{H} 10 \mathrm{~B}$ & 0.9800 \\
$\mathrm{O} 1-\mathrm{C} 7$ & $1.377(2)$ & $\mathrm{C} 10-\mathrm{H} 10 \mathrm{C}$ & 0.9800
\end{tabular}




\begin{tabular}{|c|c|c|c|}
\hline $\mathrm{C} 1-\mathrm{C} 8$ & $1.362(2)$ & $\mathrm{C} 11-\mathrm{C} 12$ & $1.387(2)$ \\
\hline $\mathrm{C} 1-\mathrm{C} 2$ & $1.448(2)$ & $\mathrm{C} 11-\mathrm{C} 16$ & $1.391(2)$ \\
\hline $\mathrm{C} 2-\mathrm{C} 7$ & $1.395(2)$ & $\mathrm{C} 12-\mathrm{C} 13$ & $1.379(3)$ \\
\hline $\mathrm{C} 2-\mathrm{C} 3$ & $1.398(2)$ & $\mathrm{C} 12-\mathrm{H} 12$ & 0.9500 \\
\hline $\mathrm{C} 3-\mathrm{C} 4$ & $1.382(3)$ & $\mathrm{C} 13-\mathrm{C} 14$ & $1.371(3)$ \\
\hline $\mathrm{C} 3-\mathrm{H} 3$ & 0.9500 & $\mathrm{C} 13-\mathrm{H} 13$ & 0.9500 \\
\hline $\mathrm{C} 4-\mathrm{C} 5$ & $1.392(3)$ & $\mathrm{C} 14-\mathrm{C} 15$ & $1.371(3)$ \\
\hline $\mathrm{C} 5-\mathrm{C} 6$ & $1.389(2)$ & $\mathrm{C} 15-\mathrm{C} 16$ & $1.385(3)$ \\
\hline $\mathrm{C} 5-\mathrm{H} 5$ & 0.9500 & $\mathrm{C} 15-\mathrm{H} 15$ & 0.9500 \\
\hline $\mathrm{C} 6-\mathrm{C} 7$ & $1.383(2)$ & $\mathrm{C} 16-\mathrm{H} 16$ & 0.9500 \\
\hline $\mathrm{O} 2-\mathrm{S} 1-\mathrm{O} 3$ & $119.77(8)$ & $\mathrm{C} 6-\mathrm{C} 9-\mathrm{H} 9 \mathrm{~A}$ & 109.5 \\
\hline $\mathrm{O} 2-\mathrm{S} 1-\mathrm{C} 1$ & $106.57(8)$ & $\mathrm{C} 6-\mathrm{C} 9-\mathrm{H} 9 \mathrm{~B}$ & 109.5 \\
\hline $\mathrm{O} 3-\mathrm{S} 1-\mathrm{C} 1$ & $108.82(8)$ & $\mathrm{H} 9 \mathrm{~A}-\mathrm{C} 9-\mathrm{H} 9 \mathrm{~B}$ & 109.5 \\
\hline $\mathrm{O} 2-\mathrm{S} 1-\mathrm{C} 11$ & $107.04(8)$ & $\mathrm{C} 6-\mathrm{C} 9-\mathrm{H} 9 \mathrm{C}$ & 109.5 \\
\hline $\mathrm{O} 3-\mathrm{S} 1-\mathrm{C} 11$ & $107.58(8)$ & $\mathrm{H} 9 \mathrm{~A}-\mathrm{C} 9-\mathrm{H} 9 \mathrm{C}$ & 109.5 \\
\hline $\mathrm{C} 1-\mathrm{S} 1-\mathrm{C} 11$ & $106.33(8)$ & $\mathrm{H} 9 \mathrm{~B}-\mathrm{C} 9-\mathrm{H} 9 \mathrm{C}$ & 109.5 \\
\hline $\mathrm{C} 8-\mathrm{O} 1-\mathrm{C} 7$ & $107.04(13)$ & $\mathrm{C} 8-\mathrm{C} 10-\mathrm{H} 10 \mathrm{~A}$ & 109.5 \\
\hline $\mathrm{C} 8-\mathrm{C} 1-\mathrm{C} 2$ & $107.36(15)$ & $\mathrm{C} 8-\mathrm{C} 10-\mathrm{H} 10 \mathrm{~B}$ & 109.5 \\
\hline $\mathrm{C} 8-\mathrm{C} 1-\mathrm{S} 1$ & $126.67(13)$ & $\mathrm{H} 10 \mathrm{~A}-\mathrm{C} 10-\mathrm{H} 10 \mathrm{~B}$ & 109.5 \\
\hline $\mathrm{C} 2-\mathrm{C} 1-\mathrm{S} 1$ & $125.96(13)$ & $\mathrm{C} 8-\mathrm{C} 10-\mathrm{H} 10 \mathrm{C}$ & 109.5 \\
\hline $\mathrm{C} 7-\mathrm{C} 2-\mathrm{C} 3$ & $119.41(15)$ & $\mathrm{H} 10 \mathrm{~A}-\mathrm{C} 10-\mathrm{H} 10 \mathrm{C}$ & 109.5 \\
\hline $\mathrm{C} 7-\mathrm{C} 2-\mathrm{C} 1$ & $104.63(14)$ & $\mathrm{H} 10 \mathrm{~B}-\mathrm{C} 10-\mathrm{H} 10 \mathrm{C}$ & 109.5 \\
\hline $\mathrm{C} 3-\mathrm{C} 2-\mathrm{C} 1$ & $135.96(16)$ & $\mathrm{C} 12-\mathrm{C} 11-\mathrm{C} 16$ & $121.12(16)$ \\
\hline $\mathrm{C} 4-\mathrm{C} 3-\mathrm{C} 2$ & $115.86(16)$ & $\mathrm{C} 12-\mathrm{C} 11-\mathrm{S} 1$ & $119.34(13)$ \\
\hline $\mathrm{C} 4-\mathrm{C} 3-\mathrm{H} 3$ & 122.1 & $\mathrm{C} 16-\mathrm{C} 11-\mathrm{S} 1$ & $119.49(13)$ \\
\hline $\mathrm{C} 2-\mathrm{C} 3-\mathrm{H} 3$ & 122.1 & $\mathrm{C} 13-\mathrm{C} 12-\mathrm{C} 11$ & $119.55(17)$ \\
\hline $\mathrm{C} 3-\mathrm{C} 4-\mathrm{C} 5$ & $123.81(16)$ & $\mathrm{C} 13-\mathrm{C} 12-\mathrm{H} 12$ & 120.2 \\
\hline $\mathrm{C} 3-\mathrm{C} 4-\mathrm{Br} 1$ & $118.15(14)$ & $\mathrm{C} 11-\mathrm{C} 12-\mathrm{H} 12$ & 120.2 \\
\hline $\mathrm{C} 5-\mathrm{C} 4-\mathrm{Br} 1$ & $118.04(13)$ & $\mathrm{C} 14-\mathrm{C} 13-\mathrm{C} 12$ & $118.25(18)$ \\
\hline $\mathrm{C} 6-\mathrm{C} 5-\mathrm{C} 4$ & $121.09(17)$ & $\mathrm{C} 14-\mathrm{C} 13-\mathrm{H} 13$ & 120.9 \\
\hline $\mathrm{C} 6-\mathrm{C} 5-\mathrm{H} 5$ & 119.5 & $\mathrm{C} 12-\mathrm{C} 13-\mathrm{H} 13$ & 120.9 \\
\hline $\mathrm{C} 4-\mathrm{C} 5-\mathrm{H} 5$ & 119.5 & $\mathrm{~F} 1-\mathrm{C} 14-\mathrm{C} 13$ & $118.26(19)$ \\
\hline $\mathrm{C} 7-\mathrm{C} 6-\mathrm{C} 5$ & $114.69(16)$ & $\mathrm{F} 1-\mathrm{C} 14-\mathrm{C} 15$ & $118.05(19)$ \\
\hline $\mathrm{C} 7-\mathrm{C} 6-\mathrm{C} 9$ & $122.24(16)$ & $\mathrm{C} 13-\mathrm{C} 14-\mathrm{C} 15$ & $123.68(18)$ \\
\hline $\mathrm{C} 5-\mathrm{C} 6-\mathrm{C} 9$ & $123.06(17)$ & $\mathrm{C} 14-\mathrm{C} 15-\mathrm{C} 16$ & $118.15(18)$ \\
\hline $\mathrm{O} 1-\mathrm{C} 7-\mathrm{C} 6$ & $124.43(16)$ & $\mathrm{C} 14-\mathrm{C} 15-\mathrm{H} 15$ & 120.9 \\
\hline $\mathrm{O} 1-\mathrm{C} 7-\mathrm{C} 2$ & $110.43(14)$ & $\mathrm{C} 16-\mathrm{C} 15-\mathrm{H} 15$ & 120.9 \\
\hline $\mathrm{C} 6-\mathrm{C} 7-\mathrm{C} 2$ & $125.14(15)$ & $\mathrm{C} 15-\mathrm{C} 16-\mathrm{C} 11$ & $119.24(17)$ \\
\hline $\mathrm{C} 1-\mathrm{C} 8-\mathrm{O} 1$ & $110.54(15)$ & $\mathrm{C} 15-\mathrm{C} 16-\mathrm{H} 16$ & 120.4 \\
\hline $\mathrm{C} 1-\mathrm{C} 8-\mathrm{C} 10$ & $134.51(18)$ & $\mathrm{C} 11-\mathrm{C} 16-\mathrm{H} 16$ & 120.4 \\
\hline $\mathrm{O} 1-\mathrm{C} 8-\mathrm{C} 10$ & $114.95(15)$ & & \\
\hline $\mathrm{O} 2-\mathrm{S} 1-\mathrm{C} 1-\mathrm{C} 8$ & $157.48(15)$ & $\mathrm{C} 1-\mathrm{C} 2-\mathrm{C} 7-\mathrm{O} 1$ & $-0.36(18)$ \\
\hline $\mathrm{O} 3-\mathrm{S} 1-\mathrm{C} 1-\mathrm{C} 8$ & $27.03(18)$ & $\mathrm{C} 3-\mathrm{C} 2-\mathrm{C} 7-\mathrm{C} 6$ & $-1.2(3)$ \\
\hline $\mathrm{C} 11-\mathrm{S} 1-\mathrm{C} 1-\mathrm{C} 8$ & $-88.59(17)$ & $\mathrm{C} 1-\mathrm{C} 2-\mathrm{C} 7-\mathrm{C} 6$ & $178.73(16)$ \\
\hline $\mathrm{O} 2-\mathrm{S} 1-\mathrm{C} 1-\mathrm{C} 2$ & $-22.76(17)$ & $\mathrm{C} 2-\mathrm{C} 1-\mathrm{C} 8-\mathrm{O} 1$ & $0.24(19)$ \\
\hline $\mathrm{O} 3-\mathrm{S} 1-\mathrm{C} 1-\mathrm{C} 2$ & $-153.21(14)$ & $\mathrm{S} 1-\mathrm{C} 1-\mathrm{C} 8-\mathrm{O} 1$ & $-179.96(12)$ \\
\hline
\end{tabular}




$\begin{array}{llll}\mathrm{C} 11-\mathrm{S} 1-\mathrm{C} 1-\mathrm{C} 2 & 91.18(15) & \mathrm{C} 2-\mathrm{C} 1-\mathrm{C} 8-\mathrm{C} 10 & -179.02(19) \\ \mathrm{C} 8-\mathrm{C} 1-\mathrm{C} 2-\mathrm{C} 7 & 0.08(18) & \mathrm{S} 1-\mathrm{C} 1-\mathrm{C} 8-\mathrm{C} 10 & 0.8(3) \\ \mathrm{S} 1-\mathrm{C} 1-\mathrm{C} 2-\mathrm{C} 7 & -179.73(13) & \mathrm{C} 7-\mathrm{O} 1-\mathrm{C} 8-\mathrm{C} 1 & -0.46(19) \\ \mathrm{C} 8-\mathrm{C} 1-\mathrm{C} 2-\mathrm{C} 3 & 179.94(19) & \mathrm{C} 7-\mathrm{O} 1-\mathrm{C} 8-\mathrm{C} 10 & 178.96(15) \\ \mathrm{S} 1-\mathrm{C} 1-\mathrm{C} 2-\mathrm{C} 3 & 0.1(3) & \mathrm{O} 2-\mathrm{S} 1-\mathrm{C} 11-\mathrm{C} 12 & -147.79(15) \\ \mathrm{C} 7-\mathrm{C} 2-\mathrm{C} 3-\mathrm{C} 4 & 0.8(2) & \mathrm{O} 3-\mathrm{S} 1-\mathrm{C} 11-\mathrm{C} 12 & -17.86(17) \\ \mathrm{C} 1-\mathrm{C} 2-\mathrm{C} 3-\mathrm{C} 4 & -179.07(18) & \mathrm{C} 1-\mathrm{S} 1-\mathrm{C} 11-\mathrm{C} 12 & 98.59(16) \\ \mathrm{C} 2-\mathrm{C} 3-\mathrm{C} 4-\mathrm{C} 5 & 0.1(3) & \mathrm{O} 2-\mathrm{S} 1-\mathrm{C} 11-\mathrm{C} 16 & 29.83(17) \\ \mathrm{C} 2-\mathrm{C} 3-\mathrm{C} 4-\mathrm{B} 1 & 179.36(12) & \mathrm{O} 3-\mathrm{S} 1-\mathrm{C} 11-\mathrm{C} 16 & 159.77(14) \\ \mathrm{C} 3-\mathrm{C} 4-\mathrm{C} 5-\mathrm{C} 6 & -0.8(3) & \mathrm{C} 1-\mathrm{S} 1-\mathrm{C} 11-\mathrm{C} 16 & -83.78(16) \\ \mathrm{Br} 1-\mathrm{C} 4-\mathrm{C} 5-\mathrm{C} 6 & 179.98(13) & \mathrm{C} 16-\mathrm{C} 11-\mathrm{C} 12-\mathrm{C} 13 & -0.6(3) \\ \mathrm{C} 4-\mathrm{C} 5-\mathrm{C} 6-\mathrm{C} 7 & 0.5(2) & \mathrm{S} 1-\mathrm{C} 11-\mathrm{C} 12-\mathrm{C} 13 & 176.99(16) \\ \mathrm{C} 4-\mathrm{C} 5-\mathrm{C} 6-\mathrm{C} 9 & -179.85(16) & \mathrm{C} 11-\mathrm{C} 12-\mathrm{C} 13-\mathrm{C} 14 & 0.8(3) \\ \mathrm{C} 8-\mathrm{O} 1-\mathrm{C} 7-\mathrm{C} 6 & -178.59(16) & \mathrm{C} 12-\mathrm{C} 13-\mathrm{C} 14-\mathrm{F} 1 & 179.41(19) \\ \mathrm{C} 8-\mathrm{O} 1-\mathrm{C} 7-\mathrm{C} 2 & 0.51(18) & \mathrm{C} 12-\mathrm{C} 13-\mathrm{C} 14-\mathrm{C} 15 & 0.1(3) \\ \mathrm{C} 5-\mathrm{C} 6-\mathrm{C} 7-\mathrm{O} 1 & 179.48(15) & \mathrm{F} 1-\mathrm{C} 14-\mathrm{C} 15-\mathrm{C} 16 & 179.55(18) \\ \mathrm{C} 9-\mathrm{C} 6-\mathrm{C} 7-\mathrm{O} 1 & -0.2(3) & \mathrm{C} 13-\mathrm{C} 14-\mathrm{C} 15-\mathrm{C} 16 & -1.1(3) \\ \mathrm{C} 5-\mathrm{C} 6-\mathrm{C} 7-\mathrm{C} 2 & 0.5(2) & \mathrm{C} 14-\mathrm{C} 15-\mathrm{C} 16-\mathrm{C} 11 & 1.3(3) \\ \mathrm{C} 9-\mathrm{C} 6-\mathrm{C} 7-\mathrm{C} 2 & -179.19(16) & \mathrm{C} 12-\mathrm{C} 11-\mathrm{C} 16-\mathrm{C} 15 & -0.5(3) \\ \mathrm{C} 3-\mathrm{C} 2-\mathrm{C} 7-\mathrm{O} 1 & 179.75(14) & \mathrm{S} 1-\mathrm{C} 11-\mathrm{C} 16-\mathrm{C} 15 & -178.04(15) \\ & & & \end{array}$

Hydrogen-bond geometry $\left(\AA,{ }^{\circ}\right)$

\begin{tabular}{lllll}
\hline$D-\mathrm{H} \cdots A$ & $D-\mathrm{H}$ & $\mathrm{H} \cdots A$ & $D \cdots A$ & $D-\mathrm{H} \cdots A$ \\
\hline $\mathrm{C} 3-\mathrm{H} 3 \cdots \mathrm{O} 2^{\mathrm{i}}$ & 0.95 & 2.53 & $3.408(2)$ & 154 \\
$\mathrm{C} 9-\mathrm{H} 9 A \cdots \mathrm{O} 2^{\mathrm{ii}}$ & 0.98 & 2.50 & $3.304(2)$ & 139 \\
\hline
\end{tabular}

Symmetry codes: (i) $-x+1,-y+1,-z+1$; (ii) $-x+1,-y,-z+1$. 